\title{
Prediction of Maximum Moment of Rectangular Tubes Subjected to Pure Bending*
}

\author{
Kenichi MASUDA** and Dai-Heng CHEN** \\ ** Tokyo University of Science, Department of Mechanical Engineering \\ Kagurazaka 1-3, Shinjuku-ku, Tokyo 162-8601, Japan \\ E-mail: masudak@rs.kagu.tus.ac.jp
}

\begin{abstract}
In the present paper, the collapse behaviors of rectangular tubes subjected to pure bending are investigated using the finite element method. Such bending collapse has been investigated extensively. These studies have revealed the existence of two types of collapse. The first type is a collapse due to buckling at the compression flange, and the second type is a collapse due to plastic yielding at the flanges. However, another type of collapse may exist. For a rectangular tube in which the web is wider than the flange, collapse due to buckling may occur at the compression web. Furthermore, an approximate prediction method is proposed for estimating the maximum bending moment of rectangular tubes in which web buckling is also taken into account. The validity of this method is verified through comparison with the numerical results obtained by FEM under various conditions.
\end{abstract}

Key words : FEM, Pure Bending, Rectangular Tube, Buckling, Effective Width

\section{Introduction}

Rectangular and square section tubes are widely used in mechanical equipment. Therefore, a study of the collapse behaviour is important for both the design and analysis of weightefficient safety structures. Prediction of the maximum moment of square tubes subjected to pure bending was proposed by Kecman ${ }^{(1)}$, and Kecman's method ${ }^{(2)}$, as introduced in a book entitled "Energy absorption of structures and materials" (3), has been used until recently. According to Kecman's study, there are two types of collapses. The first type is a collapse due to buckling at the compression flange, and the second type is a collapse due to plastic yielding at the flanges. However, when the web is wider than the flange, collapse due to buckling at the compression web may occur, as has been reported in the bending of open-section beams ${ }^{(4)}$. In the present study, the effects of the material and geometrical properties of rectangular tubes on bending collapse are investigated using the finite element method. Furthermore, based on the obtained numerical results, a method for predicting the maximum bending moment of rectangular tubes subjected to pure bending is proposed.

In addition, the validity of the FE analysis result under bending collapse has been already verified by comparing the experimental results reported by Kyriakides ${ }^{(5)}$ with the numerical results obtained under pure bending with cylindrical tubes of aluminum alloy as reported previously by the present authors ${ }^{(6)}$.

\section{Analytical method}

The commercial FEM analysis package MSC.Marc ${ }^{(7)}$ was used to analyze the large

*Received 4 Jan., 2011 (No. T1-09-0606) Japanese Original: Trans. Jpn. Soc. Mech. Eng., Vol.76, No.761, A (2010), pp.26-33 (Received 25 June, 2009) [DOI: 10.1299/jee.6.554]

Copyright (c) 2011 by JSME elastoplastic bending of the rectangular tubes shown in Fig. 1. In the present calculation, one end of the rectangular tube was fixed to a rigid wall. Pure bending was applied from the other end by modeling a lid rotating about the $z$ axis under rotary control. The effects of various geometric parameters, such as tube thickness $t$, tube flange width $c_{1}$, and tube web width $c_{2}$, on bending collapse were investigated. The lid thickness $t_{f}$ was set to five times 
$t$, as suggested by Guarracino ${ }^{(8)}$, because the lid must be stiff enough to prevent distortion. The tube material used in the analysis was assumed to be a homogeneous and isotropic elastic perfectly plastic material that conforms to von Mises yield conditions. In the present study, the Young's modulus $E$ was assumed to be $72.4 \mathrm{GPa}$, and Poisson's ratio $v$ was assumed to be 0.3. The influence of the material properties on the bending collapse of the rectangular tube was investigated in terms of the yield stress $\sigma_{Y}$.

In the present study, the updated Lagrange method was used to formulate the geometric nonlinear behavior, and the algorithm based on the Newton-Raphson method and the returnmapping method were used to solve the nonlinear equation. The rectangular tubes were modeled using four-node quadrilateral thickness shell elements (Element type 75). The elements divided the flange and the web width into 20 sub-lengths and divided the axial length such that the elements become almost square.

In addition, the rectangle length used in the analysis was assumed to be large enough to neglect the influence of the boundary conditions. The ratio of the length and flange width $L / c_{1}$ was set to $L / c_{1}>6$.
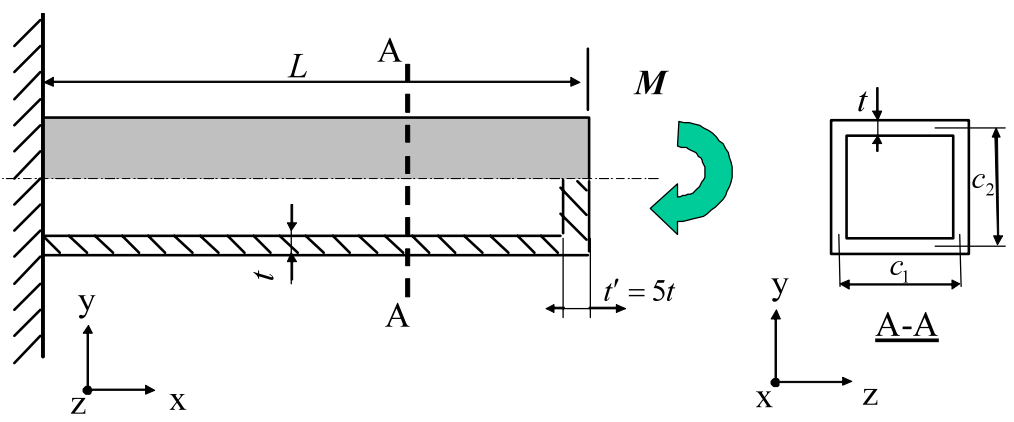

Fig. 1 Analyzed model of a rectangular tube subjected to pure bending

\section{Results and discussion}

\subsection{Comparison between the method proposed by Kecman and the results of the present} numerical analyses

First, we present Kecman's method for predicting the maximum bending moment of rectangular tubes subjected to pure bending. For a rectangular tube subjected to pure bending, the buckling stress $\sigma_{b u c}$ of the compression flange was derived as follows:

$$
a_{e}=a\left(0.7 \frac{\sigma_{b u c}}{\sigma_{Y}}+0.3\right)
$$

where $E, v, a, b$, and $t$ are Young's modulus, Poisson's ratio, the flange width, the web width, and tube thickness, respectively. In addition, $a$ is $c_{1}+t$, and $b$ is $c_{2}+t$.

Kecman proposed a method that considered three stress distribution cases using a comparison between the buckling stress $\sigma_{b u c}$ and the yield stress $\sigma_{Y}$.

(1) For the case in which $\sigma_{b u c}<\sigma_{Y}$

If the buckling stress $\sigma_{b u c}$ is less than the yield stress $\sigma_{Y}$, then the compression flange buckles, and the edges stress increases to the yield stress $\sigma_{Y}$. In order to consider this phenomenon, the effective width $a_{e}$ is introduced to the following simplified equation:

$$
a_{e}=a\left(0.7 \frac{\sigma_{b u c}}{\sigma_{Y}}+0.3\right)
$$

As a result, the stress distribution in the maximum moment is shown in Fig. 2(a). In the figure, $y_{1}$ is the distance from the compression flange to the neutral axis as derived from the condition of zero axial load. Therefore, $y_{1}$ is given as follows:

$$
\frac{y_{1}}{b}=\frac{a+b}{a_{e}+a+2 b}
$$




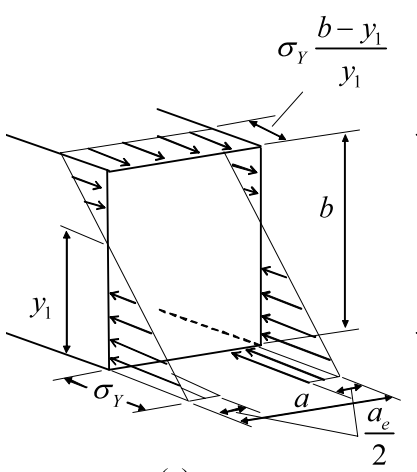

(a)

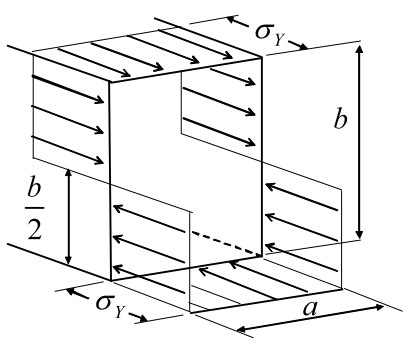

(b)

Fig. 2 Schematic representation of the axial stress distribution proposed by $\operatorname{Kecman}^{(1)}$ : (a) $\sigma_{\text {buc }}<\sigma_{Y}$; (b) $\sigma_{\text {buc }} \geq 2 \sigma_{Y}$

By summing the moments through the cross-section, the maximum bending moment is derived as follows:

$$
M_{\max }=\sigma_{Y} \cdot t \cdot b^{2} \cdot \frac{2 a+b+a_{e} \cdot\left(3 \frac{a}{b}+2\right)}{3(a+b)}
$$

(2) For the case in which $\sigma_{b u c} \geq 2 \sigma_{Y}$

In this case, the stress distribution in the maximum moment is shown in Fig. 2(b). Namely, it is assumed that the maximum moment is equal to a fully plastic moment $M_{p}$. The maximum bending moment is derived as follows:

$$
M_{\max }=M_{p}=\sigma_{Y} \cdot t\left[a(b-t)+0.5(b-2 t)^{2}\right]
$$

(3) For the case in which $\sigma_{Y} \leq \sigma_{b u c}<2 \sigma_{Y}$

First, if the buckling stress $\sigma_{b u c}$ is equal to the yield stress $\sigma_{Y}$, it is assumed that the maximum moment is equal to an elastic moment $M_{e}$, in which the stress of the flanges is equal to the yield stress $\sigma_{Y}$. This elastic moment $M_{e}$ is derived as follows:

$$
M_{e}=\sigma_{Y} \cdot t \cdot b \cdot\left(a+\frac{b}{3}\right)
$$

Finally, for the case in which $\sigma_{Y} \leq \sigma_{b u c}<2 \sigma_{Y}$, the maximum bending moment is derived by linear interpolation:

$$
M_{\max }=M_{e}+\left(M_{p}-M_{e}\right) \frac{\sigma_{b u c}-\sigma_{Y}}{\sigma_{Y}}
$$

Figure 3 compares the method proposed by Kecman and the results of the present numerical analyses for high and low aspect ratios $c_{2} / c_{1}$ with $c_{1}=50 \mathrm{~mm}, L=300 \mathrm{~mm}$, and $\sigma_{Y} / E$ $=0.001$. As shown in the figure, for high aspect ratios for which the web is wider than the flange, the maximum moment obtained under various values of $t / c_{1}$ using Kecman's method and the FEM results have the large error. In particular, the error increases with decreasing $t / c_{1}$. Basically, a region to which Kecman's method does not apply is found to exist. Therefore, it is important to reveal the bending collapse mechanism of rectangular tubes in order to predict the maximum moment.

\subsection{Two types of collapse mechanism reported by Kecman}

The two types of collapse mechanisms reported by Kecman were investigated using square tubes having an aspect ratio $c_{2} / c_{1}$ of 1 . Figure 4 shows the relationship between tube curvature $\kappa=\theta / L$ and moment $M$ for a square tube with $t=0.9 \mathrm{~mm}, c_{1}=50 \mathrm{~mm}, c_{2}=50 \mathrm{~mm}$, 


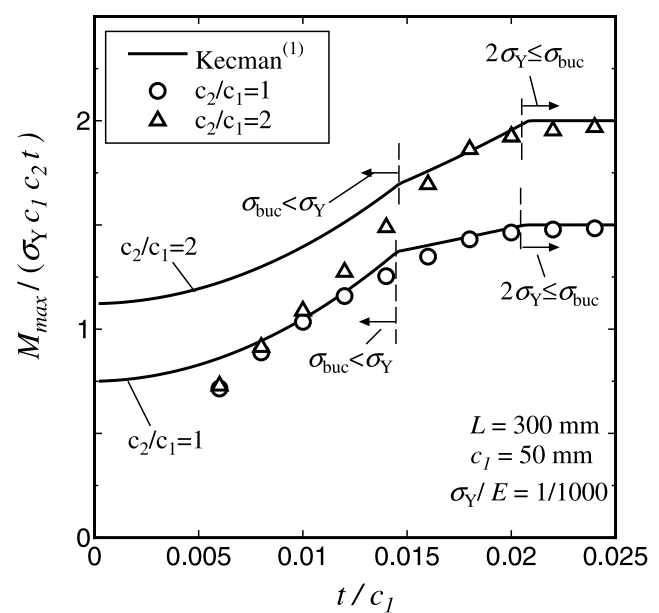

Fig. 3 Comparison of Kecman's results and the FEM results for the relationship between $t / c_{1}$ and $M_{\max } /\left(\sigma_{Y} c_{1} c_{2} t\right)$

and $\sigma_{Y} / E=0.001\left(\sigma_{b u c}=1.52 \sigma_{Y}\right)$. In addition, Fig. 4 shows the relationship between tube curvature $\kappa=\theta / L$ and axial stress $\sigma_{x}$ at points B and C (refer to the schematic representation of the tube cross-section in Fig. 4). As shown in the figure, the maximum moment is in good agreement with the value obtained using Equation (7). The axial compression stress $\sigma_{x} / \sigma_{Y}$ at point $\mathrm{B}$ in the middle of the compression flange increases until the moment becomes the maximum moment and $\sigma_{x} / \sigma_{Y}$ becomes 1 . In addition, the axial compression stress $\sigma_{x} / \sigma_{Y}$ at point $\mathrm{C}$ at the quarter-web width increases until the moment becomes the maximum moment. Figure 5 shows the axial stress distribution of the cross-section at phases $(\alpha)$ and $(\beta)$ corresponding to $\theta / L=0.025 \mathrm{~m}^{-1}$ and $0.065 \mathrm{~m}^{-1}$ in Fig. 4. As shown in the figure, the absolute value of the axial stress when the maximum moment occurs is greater than the value at phase $(\alpha)$ for all cross-sectional positions. In addition, the axial stress distribution when the maximum moment occurs is in good agreement with the results obtained by Kecman's method. The above investigation confirms that, for the case in which $c_{2} / c_{1}=1$ and $\sigma_{Y} \leq \sigma_{\text {buc }}$, the collapse is not due to buckling at the compression flange and the web, but rather plastic yielding at the flanges. Therefore, the maximum moment can be predicted by Kecman's theory in this case.

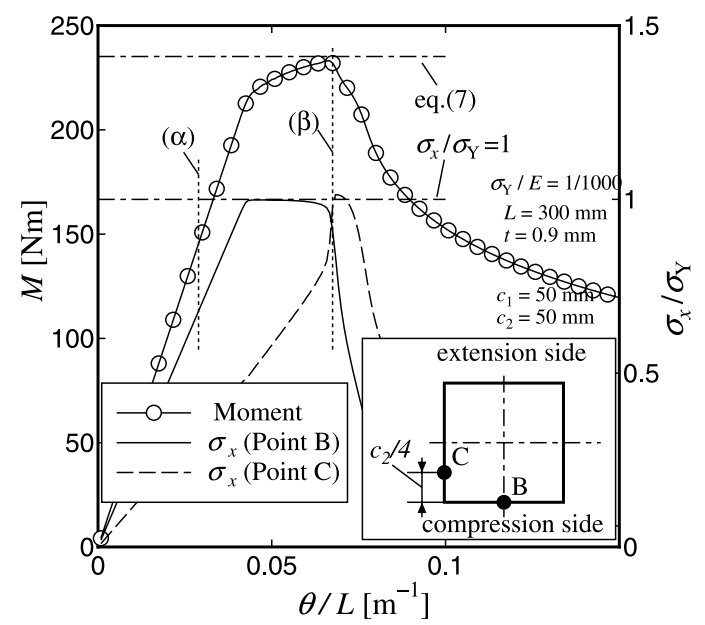

Fig. 4 Relationship between $\theta / L$ and $M, \sigma_{x} / \sigma_{Y}$ for a square tube with $t=0.9 \mathrm{~mm}, c_{1}$ $=50 \mathrm{~mm}$ and $c_{2}=50 \mathrm{~mm}$

Figure 6 shows the relationship between tube curvature $\kappa=\theta / L$ and moment $M$ for a square tube with $t=0.4 \mathrm{~mm}, c_{1}=50 \mathrm{~mm}, c_{2}=50 \mathrm{~mm}$, and $\sigma_{Y} / E=0.001\left(\sigma_{b u c}=0.31 \sigma_{Y}\right)$. In addition, Fig. 6 shows the relationship between tube curvature $\kappa=\theta / L$ and axial stress 


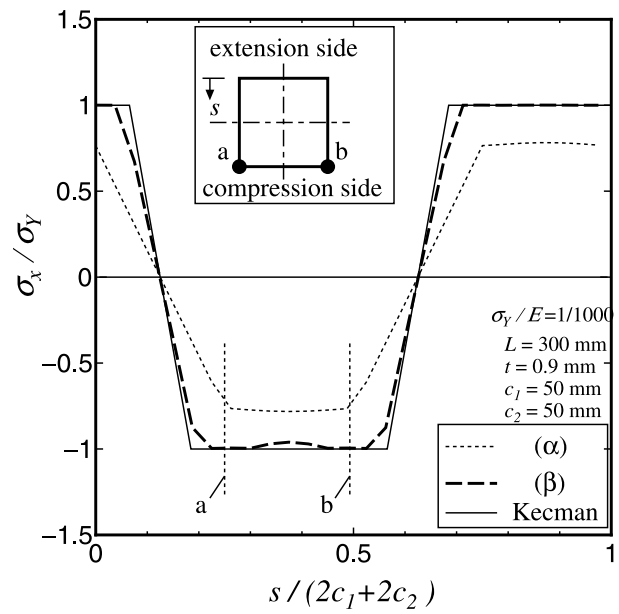

Fig. 5 Axial stress distribution of the collapse cross-section of the square tube shown in Fig.4

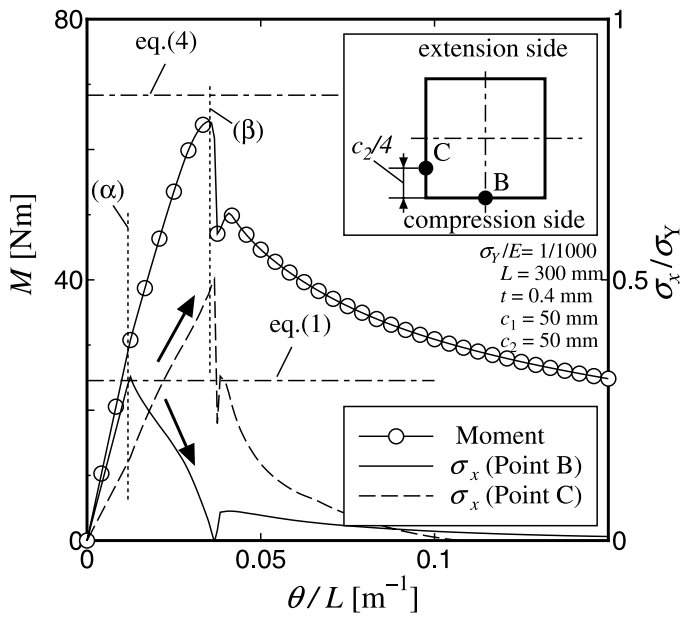

Fig. 6 Relationship between $\theta / L$ and $M, \sigma_{x} / \sigma_{Y}$ for a square tube with $t=0.4 \mathrm{~mm}, c_{1}$ $=50 \mathrm{~mm}, c_{2}=50 \mathrm{~mm}$

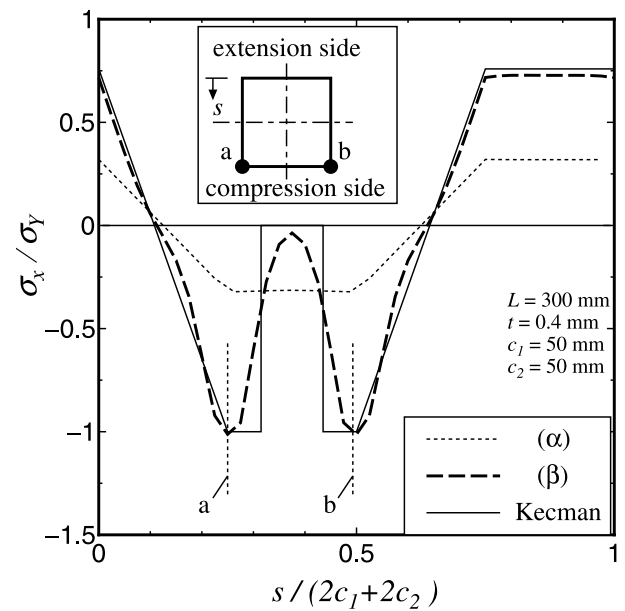

Fig. 7 Axial stress distribution of the collapse cross-section of the square tube shown in Fig.6 
$\sigma_{x}$ at points B and $\mathrm{C}$ (refer to the schematic representation of the cross-section in Fig. 6). As shown in the figure, the maximum moment is in good agreement with the value obtained using Equation (4). The axial compression stress $\sigma_{x} / \sigma_{Y}$ at point $\mathrm{B}$ in the middle of the compression flange decreases before the moment becomes the maximum moment, and the maximum value $\sigma_{x} / \sigma_{Y}$ is in good agreement with the elastic buckling stress given by Equation (1). In addition, the axial compression stress $\sigma_{x} / \sigma_{Y}$ at point $\mathrm{C}$ at the quarter-web width increases until the moment becomes the maximum moment. Figure 7 shows the axial stress distribution of the cross-section at phases $(\alpha)$ and $(\beta)$ corresponding to $\theta / L=0.012 \mathrm{~m}^{-1}$ and $0.038 \mathrm{~m}^{-1}$, respectively, in Fig. 6. As shown in the figure, although the axial compression stress in the middle of the compression flange decreases due to buckling in the middle of the compression flange, the axial compression stress increases at both edges of the compression flange because buckling does not occur at the edges of the flange. Just after buckling, the stress increment at both edges is greater than the stress decrement in the middle of the compression flange. Therefore, the total force on the compression side increases and the moment increases. In addition, the stress distribution of the web changes linearly because buckling does not occur in the web. Therefore, the axial stress distribution when the maximum moment occurs is in good agreement with that obtained by Kecman's method using the effective width of the compression flange. The above investigation confirms that for the case in which $c_{2} / c_{1}=1$ and $\sigma_{Y}>\sigma_{b u c}$, collapse is due to buckling at the compression flange. Therefore, the maximum moment can be predicted by Kecman's theory in this case.

\subsection{Collapse mechanism which is different from Kecman's indication}

In the case of a high aspect ratio, for which the web is wider than the flange, collapse was confirmed to be due to buckling at the compression web. Figure 8 shows the relationship between tube curvature $\kappa=\theta / L$ and moment $M$ for a rectangular tube with $t=0.5 \mathrm{~mm}, c_{1}=$ $20 \mathrm{~mm}, c_{2}=100 \mathrm{~mm}$, and $\sigma_{Y} / E=0.001\left(\sigma_{b u c}=2.83 \sigma_{Y}, c_{2} / c_{1}=5\right)$. In addition, Fig. 8 also shows the relationship between tube curvature $\kappa=\theta / L$ and axial stress $\sigma_{x}$ at points B and C (refer to the schematic representation of the cross-section in Fig. 8). As shown in the figure, the maximum moment is less than that obtained using Equation (5). In addition, the axial compression stress $\sigma_{x} / \sigma_{Y}$ at point $\mathrm{B}$ in the middle of the compression flange increases until the moment becomes the maximum moment and the value $\sigma_{x} / \sigma_{Y}$ becomes 1 . In addition, the axial compression stress $\sigma_{x} / \sigma_{Y}$ at point $\mathrm{C}$ at the quarter-web width decreases before the moment becomes the maximum moment. Figure 9 shows the axial stress distribution of the cross-section at phases $(\alpha)$ and $(\beta)$, corresponding to $\theta / L=0.036 \mathrm{~m}^{-1}$ and $0.048 \mathrm{~m}^{-1}$ in Fig. 8. As shown in the figure, the axial stress distribution in the compression flange is constant, and the absolute value is approximately 1 when the maximum moment occurs. In addition, the axial stress distribution in the compression web does not increase linearly. Therefore, the sum of the axial stresses when the maximum moment occurs is less than that obtained by Kecman's method, as indicated by the arrows in Fig. 9. The above investigation reveals that, in the case of a high aspect ratio and $\sigma_{Y}<\sigma_{b u c}$, collapse is not due to plastic yielding at the flanges, but rather buckling at the compression web in a state of plastic yielding at the compression flange. Therefore, the maximum moment cannot be predicted by Kecman's theory in this case.

Figure 10 shows the relationship between tube curvature $\kappa=\theta / L$ and moment $M$ for a rectangular tube with $t=0.4 \mathrm{~mm}, c_{1}=50 \mathrm{~mm}, c_{2}=100 \mathrm{~mm}$, and $\sigma_{Y} / E=0.001\left(\sigma_{b u c}=\right.$ $\left.0.30 \sigma_{Y}, c_{2} / c_{1}=2\right)$. In addition, Fig. 10 shows the relationship between tube curvature $\kappa=\theta / L$ and axial stress $\sigma_{x}$ at points B and C (refer to the schematic representation of the cross-section in Fig. 10). As shown in the figure, the maximum moment is less than the value obtained using Equation (4). The axial compression stress $\sigma_{x} / \sigma_{Y}$ at point B in the middle of the compression flange decreases before the moment becomes the maximum moment, and the maximum value $\sigma_{x} / \sigma_{Y}$ is in good agreement with the elastic buckling stress given by Equation (1). In addition, the axial compression stress $\sigma_{x} / \sigma_{Y}$ at point $\mathrm{C}$ at the quarter-web width decreases before the moment becomes the maximum moment. Figure 11 shows the axial 


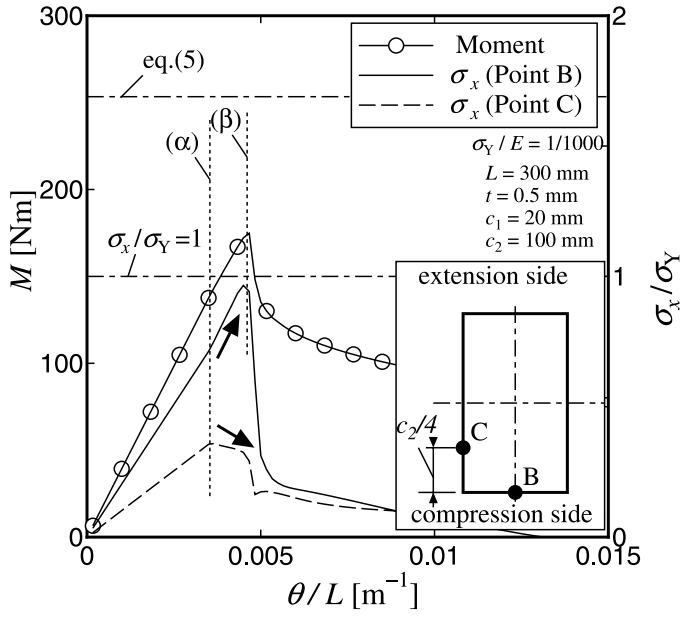

Fig. 8 Relationship between $\theta / L$ and $M, \sigma_{x} / \sigma_{Y}$ for a rectangular tube with $t=0.5 \mathrm{~mm}$, $c_{1}=20 \mathrm{~mm}$ and $c_{2}=100 \mathrm{~mm}$

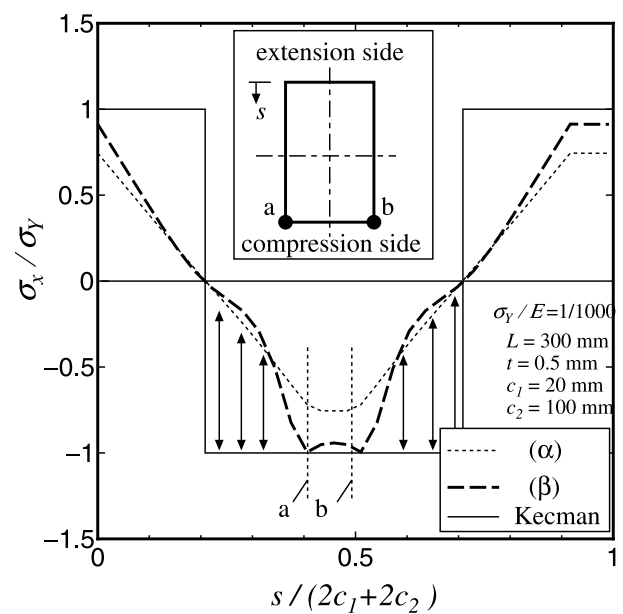

Fig. 9 Axial stress distribution of the collapse cross-section of the rectangular tube shown in Fig.8

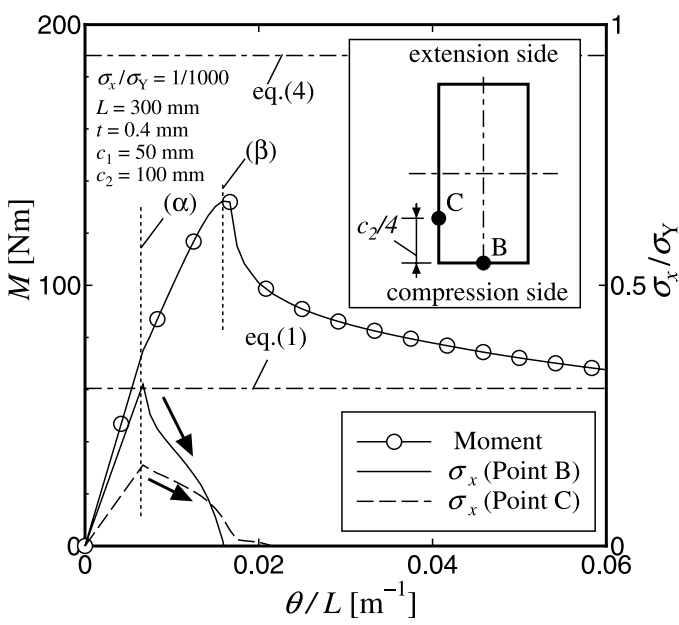

Fig. 10 Relationship between $\theta / L$ and $M, \sigma_{x} / \sigma_{Y}$ for a rectangular tube with $t=$ $0.5 \mathrm{~mm}, c_{1}=20 \mathrm{~mm}, c_{2}=100 \mathrm{~mm}$ 


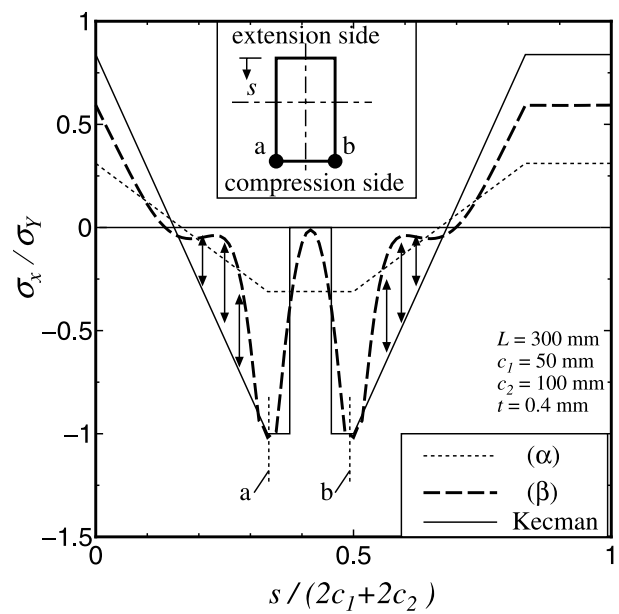

Fig. 11 Axial stress distribution of the collapse cross-section of the rectangular tube shown in Fig.10

stress distribution of the cross-section at phases $(\alpha)$ and $(\beta)$, corresponding to $\theta / L=0.007$ $m^{-1}$ and $0.016 m^{-1}$ in Fig. 10. As shown in the figure, the axial stress in the compression flange is concentrated at the edges when the maximum moment occurs. In addition, the axial stress distribution in the compression web does not increase linearly. Therefore, the sum of the axial stresses at the maximum moment is less than that obtained by Kecman's method, as indicted by the arrows in Fig. 11. The reason for this is that Equation (2) applies to the axial stress distribution of the compression flange, and linearly approximation does not apply to the axial stress distribution of the compression web in this case. The above investigation reveals that, in the case of a high aspect ratio and $\sigma_{Y} \geq \sigma_{b u c}$, the collapse is not only due to buckling at the compression flange but also due to buckling at the compression web. Therefore, the maximum moment cannot be predicted by Kecman's theory in this case.

\subsection{Proposed method of maximum moment considering web buckling}

Figure 12 shows a schematic representation of the axial stress distribution at the maximum moment when the compression flange buckles $\left(\sigma_{b u c}<\sigma_{Y}\right)$. Figure 12(a) shows the distribution of Kecman's method, which does not consider web buckling, and Fig. 12(b) shows the distribution of the proposed method, which considers web buckling. As shown in the figure, an effective width $a_{e}$ is applied to the compression web as well as the compression flange. Referring to Karman's theory ${ }^{(8)}$, the effective width $a_{e}$ is assumed to be independent of the initial web width. Equation (3) of Kecman is assumed to be applicable to the position of the neutral axis $y_{1}$ in the proposed method. The coefficient $\alpha$, which represents the axial tension stress, is derived under the condition in which the total force of the compression side and the tension side is zero. Thus,

$$
\alpha=\frac{2\left(a_{e}-t\right)}{a+b-y_{1}-2 t}
$$

is derived from the total force of the compression side, $2 \sigma_{Y} t\left(a_{e}-t\right)$, and the total force of the tension side, $\alpha \sigma_{Y} t(a-2 t)+\alpha \sigma_{Y} t\left(b-y_{1}\right)$.

Figure 13 compares the FEM results and the results obtained by Kecman's method and the proposed method for the axial stress distribution for a rectangular tube with $t=0.4 \mathrm{~mm}, c_{1}$ $=50 \mathrm{~mm}$, and $\sigma_{Y} / E=0.001$. As shown in the figure, for the high aspect ratios, i.e., $c_{2} / c_{1}=2$ and $c_{2} / c_{1}=1.6$, the results obtained by the proposed method, which considers web buckling, are in good agreement with the FEM results. For the low aspect ratio $c_{2} / c_{1}=0.6$, the results obtained by Kecman's method, which does not consider web buckling, are in good agreement with the FEM results.

Figure 14 shows a schematic representation of the axial stress distribution at the maxi- 


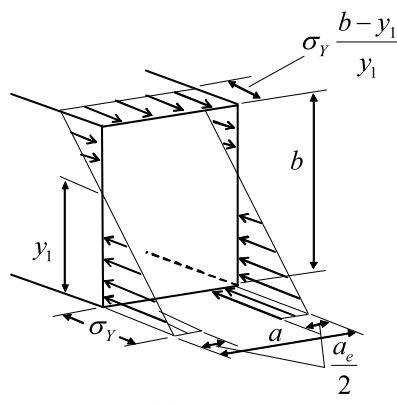

(a)

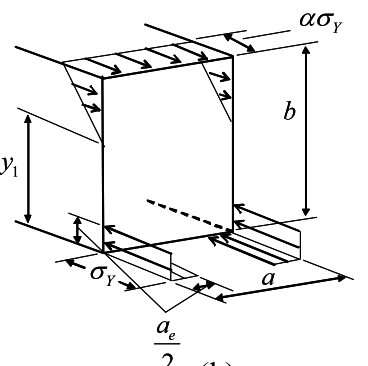

(b)

Fig. 12 Axial stress distribution in the range of $\sigma_{b u c}<\sigma_{Y}$ as obtained (a) by Kecman's method and (b) by the proposed method

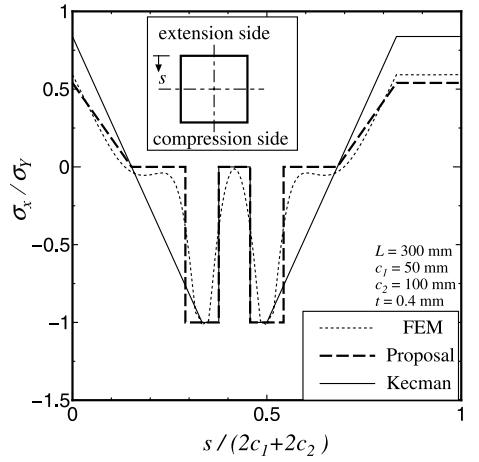

(a)

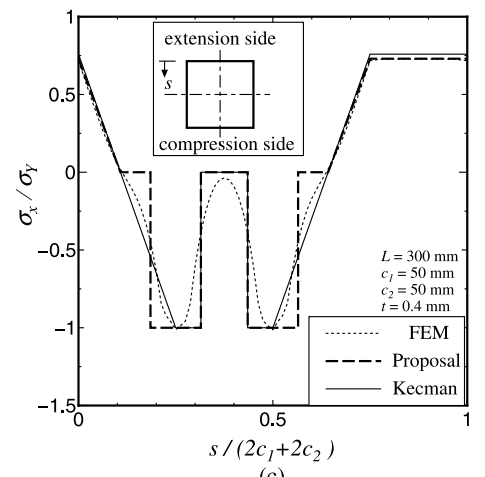

(c)

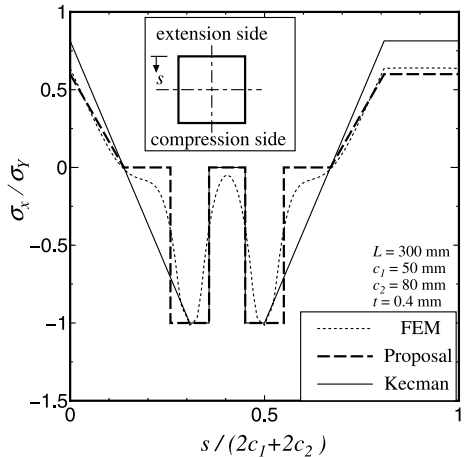

(b)

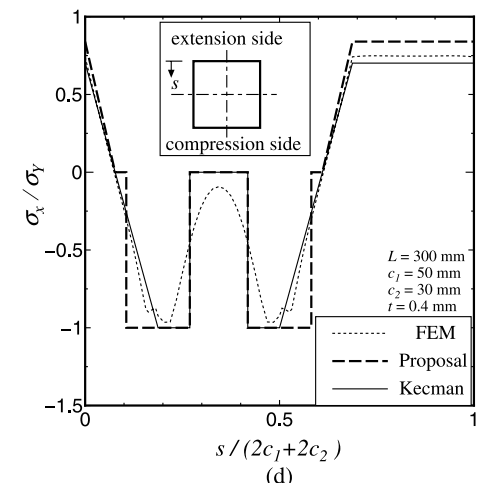

(d)

Fig. 13 Axial stress distribution obtained by the FEM, Kecman's method, and the proposed method for (a) $c_{2} / c_{1}=2$, (b) $c_{2} / c_{1}=1.6$, (c) $c_{2} / c_{1}=1$, and (d) $c_{2} / c_{1}=0.6$ 
mum moment when the compression flange does not buckle $\left(\sigma_{b u c} \geq \sigma_{Y}\right)$. Figure 14(a) shows Kecman's results, which do not consider web buckling, and Fig. 14(b) shows the results obtained by the proposed method, which considers web buckling. As in the case of Fig. 12(b), Equation (2) applies to the effective width $a_{e}$ in the compression web, and Equation (3) applies to the position of the neutral axis $y_{1}$ in the proposed method. The coefficient $\beta$, which represents the axial tension stress, is derived under the condition in which the total force of the compression side and the tension side is zero. Thus,

$$
\beta=\frac{a+a_{e}-2 t}{a+b-y_{1}-2 t}
$$

is derived from the total force of the compression side, $\sigma_{Y} t(a-2 t)+\sigma_{Y} t a_{e}$, and the total force of the tension side, $\beta \sigma_{Y} t(a-2 t)+\beta \sigma_{Y} t\left(b-y_{1}\right)$.

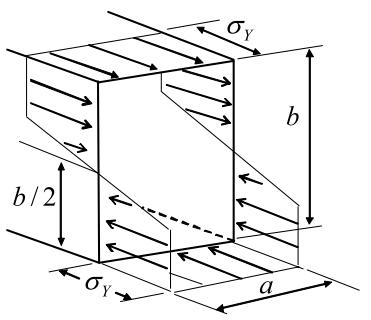

(a)

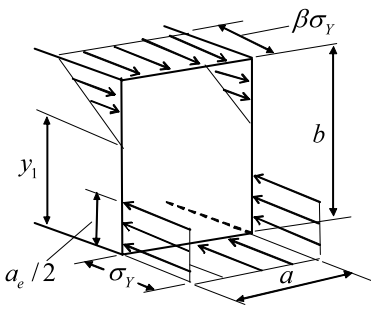

(b)

Fig. 14 Axial stress distribution in the range of $\sigma_{b u c} \geq \sigma_{Y}$ as obtained (a) by Kecman's method and (b) by the proposed method

Figures 15(a) and 15(b) compare the FEM results and the results obtained by Kecman's method and the proposed method for the axial stress distribution for a rectangular tube with $t=0.5 \mathrm{~mm}, c_{1}=20 \mathrm{~mm}$, and $\sigma_{Y} / E=0.001$. As shown in the figures, at a high aspect ratio, $c_{2} / c_{1}=5$, the results of the proposed method, which considers web buckling, are in good agreement with the FEM results. At a low aspect ratio, $c_{2} / c_{1}=2$, the results obtained by Kecman's method, which does not consider web buckling, are in good agreement with the FEM results.

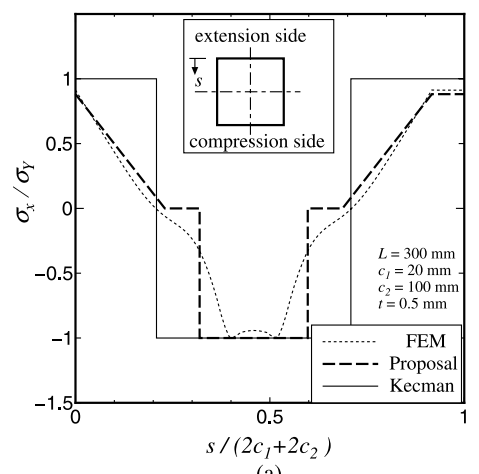

(a)

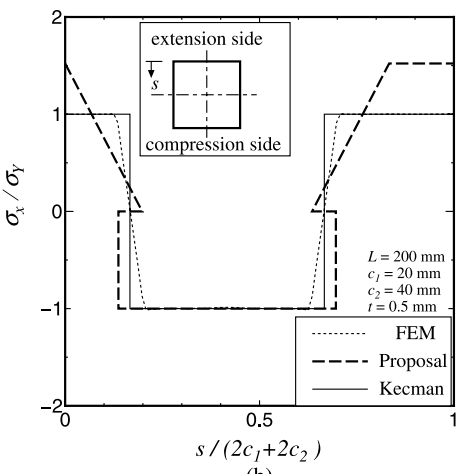

(b)

Fig. 15 Axial stress distribution obtained by the FEM results, Kecman's method, and the proposed method for (a) $c_{2} / c_{1}=5$ and (b) $c_{2} / c_{1}=2$

Finally, we can present the proposed method for predicting the maximum bending moment of rectangular tubes subjected to pure bending, because the axial stress distributions which consider web buckling have been presented. For the case in which $\sigma_{b u c}<\sigma_{Y}$, the position of the center of gravity in the tension web $G$ is derived as follows:

$$
G=\frac{1}{3}\left(\frac{1}{2} b+y_{1}\right)
$$


Therefore, for the case in which $\sigma_{b u c}<\sigma_{Y}$, the moments of the individual parts at the maximum moment are as follows. The moment of the tension web is $\frac{1}{2} \alpha \sigma_{Y} t\left(b-y_{1}\right) \times G \times 2$, and the moment of the tension flange is $\alpha \sigma_{Y} t(a-2 t) \times \frac{1}{2}(b-t)$. The moment of the compression web is $\sigma_{Y} \frac{a_{e}}{2} t \times\left(\frac{b}{2}-\frac{a_{e}}{4}\right) \times 2$, and the moment of the compression flange is $\sigma_{Y}\left(a_{e}-2 t\right) t \times \frac{1}{2}(b-t)$. Therefore, for the case in which $\sigma_{b u c}<\sigma_{Y}$, the maximum moment at which the compression web buckles is derived as follows:

$$
\begin{aligned}
M_{\max }= & \frac{1}{2} \sigma_{Y} t\left\{\alpha(a-2 t)(b-t)+2 \alpha\left(b-y_{1}\right) G\right. \\
& \left.+\left(a_{e}-2 t\right)(b-t)+2 a_{e}\left(\frac{b}{2}-\frac{a_{e}}{4}\right)\right\}
\end{aligned}
$$

where $y_{1}, \alpha$, and $G$ are obtained using Equations (3), (8), and (10), respectively. The above investigation reveals that for the case in which $\sigma_{b u c}<\sigma_{Y}$, the maximum moment is derived as follows:

$$
M_{\max }=\operatorname{Min}(e q \cdot(4), e q \cdot(11))
$$

Moreover, for the case in which $\sigma_{b u c} \geq \sigma_{Y}$, the maximum moment at which the compression web buckles is derived as follows:

$$
\begin{aligned}
M_{\max }= & \frac{1}{2} \sigma_{Y} t\left\{\beta(a-2 t)(b-t)+2 \beta\left(b-y_{1}\right) G\right. \\
& \left.+(a-2 t)(b-t)+2 a_{e}\left(\frac{b}{2}-\frac{a_{e}}{4}\right)\right\}
\end{aligned}
$$

where $y_{1}, \beta$, and $G$ are obtained using Equations (3), (9), and (10), respectively. The above investigation reveals that for the case in which $\sigma_{b u c} \geq \sigma_{Y}$, the maximum moment is derived as follows:

$$
M_{\max }=\operatorname{Min}(e q \cdot(5), e q \cdot(7), e q \cdot(13))
$$

Figure 16 compares the FEM results and the results obtained by Kecman's method and the proposed method at the maximum moment for $t=0.5 \mathrm{~mm}$ and $0.4 \mathrm{~mm}$. As shown in the figure, the lower values obtained by Kecman's method and the proposed method are in good agreement with the FEM results. Figure 17 compares the FEM results and the results obtained by Kecman's method and the proposed method at the maximum moment for $\sigma_{Y} / E$ $=1 / 1,000$, and 1/500. As shown in the figure, the lower values obtained by Kecman's method and the proposed method are in good agreement with the FEM results. Figure 18 shows the mode map for the $t / c_{1}-c_{2} / c_{1}$ relationship. The $\bullet$ points indicate that the maximum moment obtained by FEM is closer to that obtained by the proposed method than that obtained by Kecman's method. The o plots indicate that the maximum moment obtained by FEM is closer to that obtained by Kecman's method than that obtained by the proposed method. Using Kecman's method and the proposed method, Fig. 18 also indicates whether web buckling occurs. As shown in the figure, the boundary line is distributed almost exactly between the collapse modes. Moreover, the maximum moment can be predicted by the $\bullet$ points using the proposed method.

\section{Conclusion}

In the present paper, bending collapse of rectangular tubes was investigated using the finite element method. The following results were obtained.

(1) The maximum moment of a rectangular tube subjected to pure bending is associated with buckling at the compression flange, plastic yielding at the flanges, and buckling at the compression web.

(2) Using the proposed method, which applies an effective width to the web, it is possible to obtain the axial stress distribution for buckling at the compression web.

( 3 ) It is possible to predict the maximum moment and the collapse mode for various materials and geometrical properties using the proposed method and Kecman's method. 


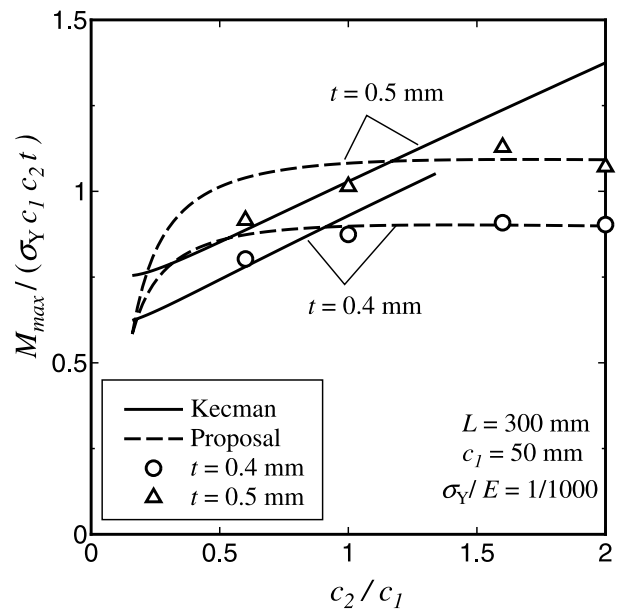

Fig. 16 Comparison between the FEM results and the results obtained by Kecman's method and the proposed method for the relationship between $c_{2} / c_{1}$ and $M_{\max } /\left(\sigma_{Y} c_{1} c_{2} t\right)$ for $t=0.5 \mathrm{~mm}$ and $0.4 \mathrm{~mm}$

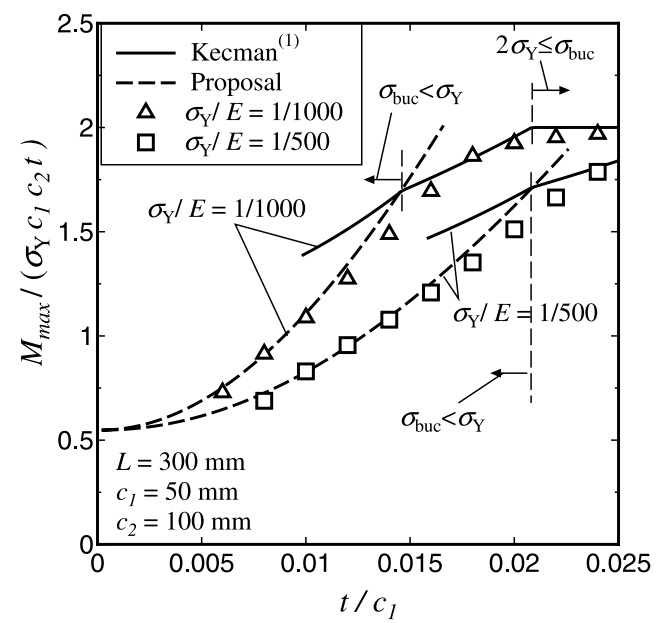

Fig. 17 Comparison of the FEM results and the results obtained by Kecman's method and the proposed method for the relationship between $t / c_{1}$ and $M_{\max } /\left(\sigma_{Y} c_{1} c_{2} t\right)$ for $\sigma_{Y} / E=1 / 1,000$, and $1 / 500$.

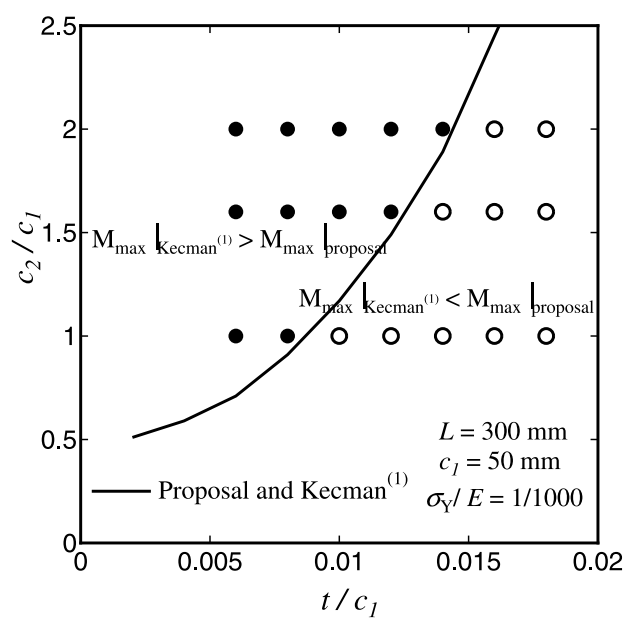

Fig. 18 Mode map in $\left(t / c_{1}, c_{2} / c_{1}\right)$ 


\section{References}

( 1 ) Kecman,D., Bending collapse of rectangular and square sectiontubes, International Journal of Mechanical Sciences, Vol.25, (1983), pp.623-36.

( 2 ) Kim, T. H., Reid, S. R., Bending collapse of thin-walled rectangular section columns, Computers and Structures, Vol.79, (2001), pp.1897-1911.

( 3 ) Lu, G., Yu, T. X., Energy absorption of structures and materials, (2003),Section 5, Crc Pr I Llc.

( 4 ) Parh, M. S. and Lee, B. C., Prediction of bending collapse behaviours of thin-walled open section beams Thin-Walled Structures, Vol.25, No.3, (1996), pp.185-206.

( 5 ) Chen, D., Masuda, K. and Ozaki, S., Study on Elastoplastic Pure Bending Collapse of Cylindrical Tubes, Transactions of the Japan Society of Mechanical Engineers, Series A, Vol.74, No.740(2008), pp.520-527.

( 6 ) Kyriakides, S. and Ju G. T., Bifurcation and localization instabilities in cylindrical shells under bending-I, II. Experiments,Predictions International Journal of Solids and Structures, Vol.29, (1992), pp.1117-1171.

( 7 ) Guarracino, F., On the analysis of cylindrical tubes under flexure: theoretical formulations, experimental data and finite element analyses, Thin-Walled Structures, Vol.41, (2003), pp.127-147.

( 8 ) Karman, V.T., Sechler, E.E. and Donnell, H.L., Strength of thin plates in compression, Trans ASME, Vol.54, (1932), pp.53-57. 